\title{
Spontaneous Remission of Epstein-Barr Virus-Positive Diffuse Large B-Cell Lymphoma of the Elderly
}

\author{
T. Mizuno ${ }^{\mathrm{a}} \quad$ M. Ishigaki ${ }^{\mathrm{a}} \quad$ K. Nakajima ${ }^{\mathrm{a}} \quad$ T. Matsue $^{\mathrm{a}} \quad$ M. Fukushima ${ }^{\text {h }}$ \\ H. Minato ${ }^{h} \quad$ N. Nojima ${ }^{h}$ Saito Atsushi ${ }^{b} \quad$ K. Ishigami $^{c} \quad H$. Atsumi ${ }^{d}$

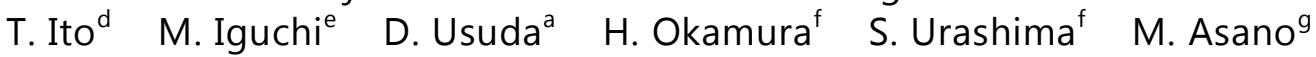 \\ A. Fukuda ${ }^{9} \quad$ Y. Izumi $^{9} \quad$ N. Takekoshi ${ }^{9} \quad$ T. Kanda ${ }^{a}$
}

Departments of a Community Medicine, ${ }^{b}$ Nephrology, ${ }^{c}$ Geriatric Medicine, ${ }^{d}$ Endocrinology,

${ }^{e}$ Respiratory Medicine, ${ }^{f}$ Gastroenterology, and ${ }^{g}$ Cardiology, Kanazawa Medical University, Himi Municipal Hospital, Himi, and hepartment of Pathology and Laboratory Medicine, Kanazawa Medical University, Ishikawa, Japan

\section{Key Words}

Elderly · Epstein-Barr virus · Remission · sIL-2R

\begin{abstract}
A 94-year-old female patient presented with anorexia and left axillar lymphadenopathy on admission. Her past history was angina pectoris at 83 years of age and total gastrectomy due to gastric cancer at 87 years. The family history revealed that her son had had a malignant lymphoma, the histopathological diagnosis of which was diffuse large B-cell lymphoma. A physical examination showed both cervical, axillar, and inguinal lymphadenopathy without tenderness. She had elevated lactate dehydrogenase, ferritin, and soluble interleukin-2 receptor (sIL-2R). Whole-body computed tomography confirmed the cervical, axillary, and inguinal lymphadenopathy. Gallium-68 imaging revealed positive accumulation in these superficial lymph nodes. A right inguinal lymph node biopsy showed features of Epstein-Barr virus-associated lymphoproliferative disorder. Immunohistological studies on this lymph node biopsy showed CD20-positive large cells, CD3-positive small cells, and CD30-partlypositive large cells. In situ hybridization showed Epstein-Barr virus-positive, LMP-partlypositive, and EBNA2-negative cells. She refused chemotherapy as her son had died from hematemesis during chemotherapy. She received intravenous hyperalimentation for 1 month after admission. No palpable lymph nodes were identified by physical examination or
\end{abstract}

\footnotetext{
T. Kanda

Department of Community Medicine

Kanazawa Medical University, Himi Municipal Hospital

1130 Kurakawa, Himi, Toyama 935-8531 (Japan)

E-Mail kandat@kanazawa-med.ac.jp
} 
Mizuno et al.: Spontaneous Remission of Epstein-Barr Virus-Positive Diffuse Large B-Cell Lymphoma of the Elderly

computed tomography 3 months after admission, and regression of lactate dehydrogenase, ferritin, and sIL-2R was observed. She recovered from anorexia and was discharged. She died from pneumonia 10 months later after initial symptoms of anorexia. The autopsy showed no superficial lymphadenopathy.

\section{Introduction}

Epstein-Barr virus (EBV)-positive diffuse large B-cell lymphoma (DLBCL) of the elderly was included in the 2008 WHO classification as a new provisional entity and is defined as blastic, clonal B-cell proliferation associated with EBV occurring in patients $>50$ years of age, presumably due to senescence of the immune system [1]. Patients with EBV-positive DLBCL of the elderly, whose clinical course is characterized by a short survival rate of approximately 24 months, were initially described by Oyama et al. [2] in 2003 in a study of $22 \mathrm{im}$ munosenescent elderly patients.

We treated a 94-year-old female who was diagnosed with EBV-positive DLBCL of the elderly. However, her lymphadenopathies regressed and her symptoms remitted completely without chemotherapy. Autopsy was performed after she died with pneumonia. We present this rare case including a review of the literature.

\section{Case Report}

A 94-year-old female patient presented with anorexia and left axillar lymphadenopathy on admission. Her past history was angina pectoris at 83 years of age and total gastrectomy due to gastric cancer at 87 years. The family history revealed that her son had had a malignant lymphoma, the histopathological diagnosis of which was DLBCL. A physical examination showed both cervical, axillar, and inguinal lymphadenopathy without tenderness. She refused chemotherapy as her son had died from hematemesis during chemotherapy. No palpable lymph nodes were identified by physical examination or computed tomography 3 months after admission, and regression of soluble interleukin-2 receptors (sIL-2R) was observed. She died from pneumonia 10 months later after initial symptoms of anorexia.

The patient had elevated ferritin, sIL-2R, and lactate dehydrogenase (LDH). These titers decreased gradually over the 10 months after admission (fig. 1). Whole-body computed tomography showed cervical, axillar, and inguinal lymphadenopathy (fig. 2), and Gallium-68 imaging revealed positive accumulation in both cervical and axillar lymph nodes (fig. 3).

Two right inguinal lymph node specimens measuring $20 \times 11 \times 8 \mathrm{~mm}$ and $10 \times 7 \times 5 \mathrm{~mm}$ were removed. After fixation in $10 \%$ buffered formalin for $24 \mathrm{~h}$, these excision biopsies were embedded in paraffin, sectioned at $4 \mu \mathrm{m}$, and stained with hematoxylin-eosin. Atypical large lymphoid cells were found with chromatin-rich nuclei and small-sized cells (fig. 4a). Immunohistological studies on this lymph node biopsy showed CD20-positive large cells, CD3-positive small cells, and CD30-partly-positive large cells (fig. 4b). In situ hybridization showed EBV-positive (fig. 4c), LMP-partly-positive, and EBNA2-negative cells. 
Mizuno et al.: Spontaneous Remission of Epstein-Barr Virus-Positive Diffuse Large B-Cell Lymphoma of the Elderly

\section{Discussion}

We have presented a case of EBV-positive DLBCL of the elderly which remitted completely without chemotherapy. No lymph nodes were palpable, and circulating LDH, ferritin, and sIL-2R were decreased after admission.

This case is unusual with respect to the patient's age and DLBCL regression. Our patient is the oldest case of EBV-positive DLBCL of the elderly in the literature [2-4]. As we have confirmed that EBV-positive DLBCL of the elderly can be identified in such very old patients, future diagnoses in elderly persons with immunosenescence should consider EBV-positive DLBCL of the elderly. Although the spontaneous regression of DLBCL is extremely rare, it has been reported previously in organs such as the tongue, ocular portion, and central nervous system [5-7]. However, to the best of our knowledge, no case of spontaneous regression of EBV-positive DLBCL of the elderly in lymph nodes has been reported.

Ferritin, sIL-2R, and LDH are all elevated in malignant lymphoma [8]. Indeed, elevation of LDH is one of the worst prognostic factors for EBV-positive DLBCL of the elderly [3]. This patient showed a decrease in circulating ferritin, sIL-2R, and LDH with regression of lymphadenopathy. As such, a regression of this type could be predicted from these data.

The pathophysiology of EBV-positive DLBCL of the elderly is considered to be related to immunosenescence, genetic factors, and EBV infection [5]. This patient's son was previously diagnosed with malignant lymphoma, with a histopathological diagnosis of DLBCL, which is negative for EBV infection. As such, genetic factors may play a more important role in the occurrence of EBV-positive DLBCL of the elderly.

Nutritional control is known to affect immunomodulation in the elderly [9]. As a result, an optimal nutritional strategy could help to maintain a healthy immune system in such patients and increase their resistance to infection and other immune-related diseases. Nutritional status can also alter immune function, which may be relevant to lymphoma genesis [10]. As one important mechanism in lymphoma genesis appears to be related to immunosenescence, EBV-positive DLBCL of the elderly may well be related to an immunologic deterioration derived from the aging process. The fact that decreased lymphadenopathy was observed after sufficient nutrition in our case may be related to the recovery of immunosenescence, thereby possibly constituting a spontaneous remission factor in this case of EBV-positive DLBCL of the elderly.

\section{Conclusions}

EBV-positive DLBCL of the elderly is rare, occurring in patients $>50$ years of age, presumably due to senescence of the immune system. Our case remitted completely without chemotherapy after nutritional control. Although spontaneous remission factors are not clear, immunomodulation in the elderly could be considered. The prognosis and management of EBV-positive DLBCL of the elderly can be changed by nutritional control.

\section{Acknowledgements}

We acknowledge the cooperation of our patient and her family. This paper is not funded by any external source. 
Mizuno et al.: Spontaneous Remission of Epstein-Barr Virus-Positive Diffuse Large B-Cell Lymphoma of the Elderly

\section{Disclosure Statement}

The authors declare that they have no competing interests.

\section{References}

1 Adam P, Bonzheim I, Fend F, et al: Epstein-Barr virus-positive diffuse large B-cell lymphomas of the elderly. Adv Anat Pathol 2011;18:349-355.

-2 Oyama T, Ichimura K, Suzuki R, et al: Senile EBV+ B-cell lymphoproliferative disorders: a clinicopathologic study of 22 patients. Am J Surg Pathol 2003;27:16-26.

-3 Oyama T, Yamamoto K, Asano N, et al: Age-related EBV-associated B-cell lymphoproliferative disorders constitute a distinct clinicopathologic group: a study of 96 patients. Clin Cancer Res 2007;13:5124-5132.

4 Shimoyama Y, Oyama T, Asano N, et al: Senile Epstein-Barr virus-associated B-cell lymphoproliferative disorders: a mini review. J Clin Exp Hematop 2006;46:1-4.

5 Tabata C, Tabata R: Possible prediction of underlying lymphoma by high sIL-2R/ferritin ratio in hemophagocytic syndrome. Ann Hematol 2012;91:63-71.

6 Pae M, Meydani SN, Wu D: The role of nutrition in enhancing immunity in aging. Aging Dis 2012;3:91-129.

7 Lu Y, Prescott J, Sullivan-Halley J, et al: Body size, recreational physical activity, and B-cell non-Hodgkin lymphoma risk among women in the California teachers study. Am J Epidemiol 2009;170:1231-1240.

-8 Tamás L, Sári E, Répássy G, et al: Spontaneous remission in localized diffuse large B-cell lymphoma. Pathol Oncol Res 2011;17:779-784.

-9 Kase S, Namba K, Jin XH, et al: Spontaneous regression of intraocular lymphoma. Ophthalmology 2012;119:1083-1084.

10 Takeishi G, Moroki K, Kawasoe T, et al: Spontaneous regression and regrowth of central nervous system lymphomatoid granulomatosis: case report. Neurol Med Chir (Tokyo) 2011;51:801-804.

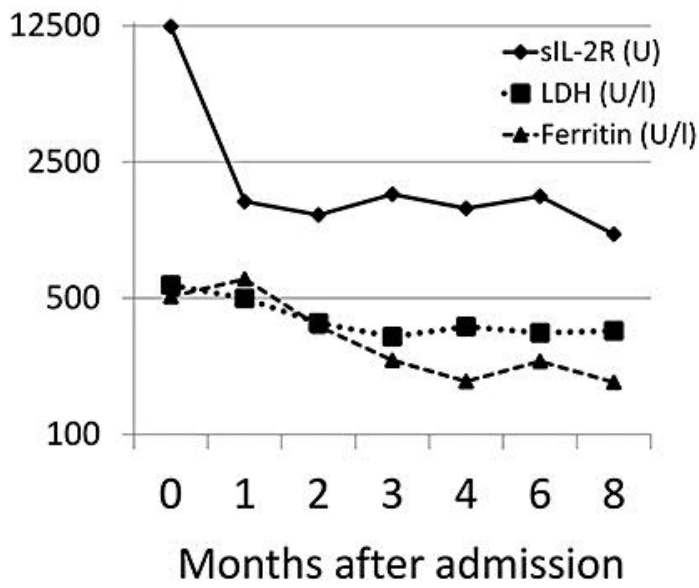

Fig. 1. Decrease of circulating sIL-2R, LDH, and ferritin with spontaneous regression of lymphadenopathy. 


\section{Case Reports in Oncology}

\begin{tabular}{l|l}
\hline \multicolumn{2}{l}{ Case Rep Oncol 2013;6:269-274 } \\
\hline DOI: $10.1159 / 000345572$ & $\begin{array}{l}\text { C 2013 S. Karger AG, Basel } \\
\text { www.karger.com/cro }\end{array}$ \\
\hline
\end{tabular}

Mizuno et al.: Spontaneous Remission of Epstein-Barr Virus-Positive Diffuse Large B-Cell Lymphoma of the Elderly

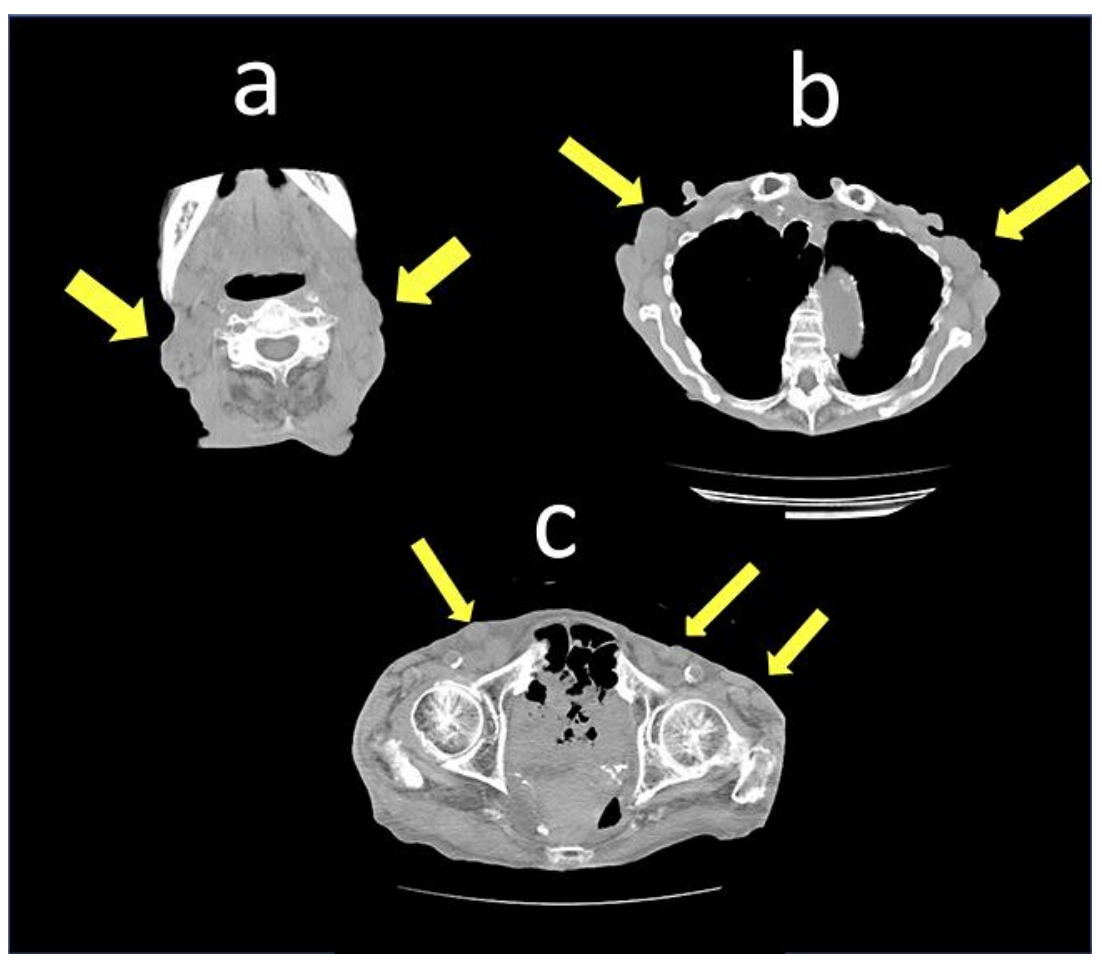

Fig. 2. Computed tomography at admission showed lymphadenopathy in the cervical, axillar, and inguinal lymph nodes.

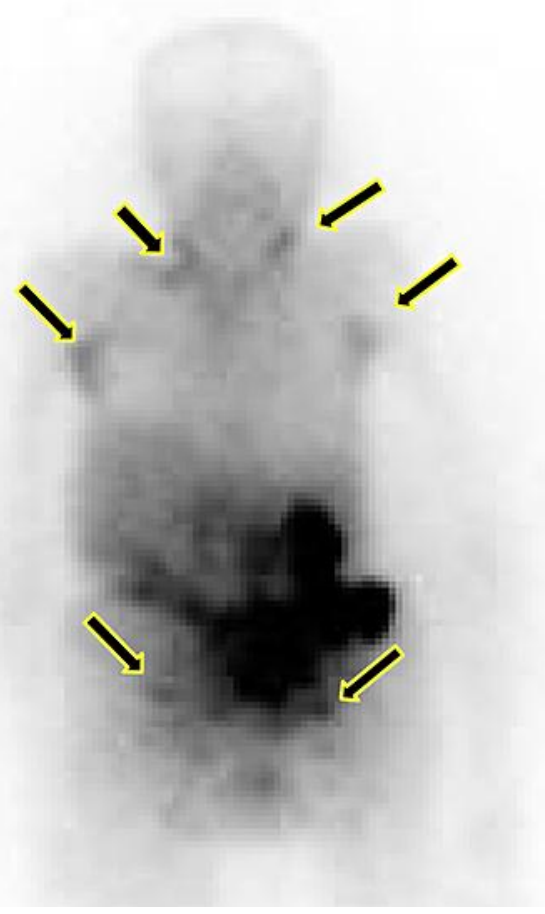

Fig. 3. Gallium-68 imaging at admission showed accumulation in superficial lymph nodes. 


\section{Case Reports in Oncology}
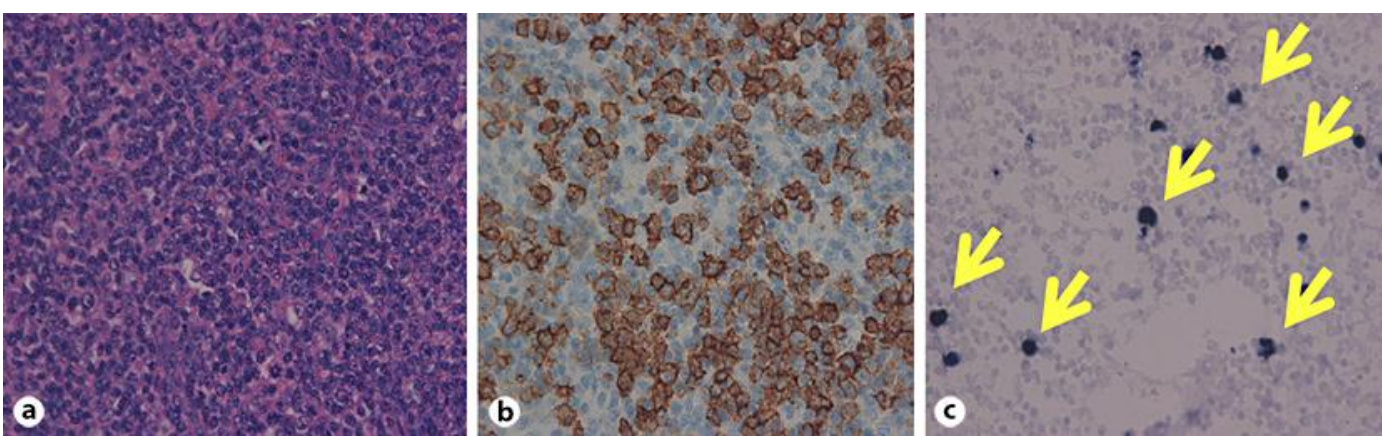

Fig. 4. Histopathological findings. a Hematoxylin-eosin staining showed atypical large cells. b Immunohistological studies on this lymph node biopsy showed CD20-positive large cells. c In situ hybridization showed EBV-positive cells (arrows). 